\title{
BANDS OF SEMIGROUPS
}

\section{A. H. CLIFFORD}

In studying a general semigroup $S$, a natural thing to do is to decompose $S$ (if possible) into the class sum of a set $\left\{S_{\alpha} ; \alpha \in I\right\}$ of mutually disjoint subsemigroups $S_{\alpha}$ such that (1) each $S_{\alpha}$ belongs to some more or less restrictive type $\mathcal{T}$ of semigroup, and (2) the product $S_{\alpha} S_{\beta}$ of any two of them is wholly contained in a third: $S_{\alpha} S_{\beta} \subseteq S_{\gamma}$, for some $\gamma \in I$ depending upon $\alpha$ and $\beta$. We shall then say that $S$ is a band of semigroups of type $\mathcal{T}$. If, for every $\alpha$ and $\beta$ in $I, S_{\alpha} S_{\beta}$ and $S_{\beta} S_{\alpha}$ are both contained in the same $S_{\gamma}$, then we shall call $S$ a semilattice of semigroups of type $\mathcal{T}$.

We shall also be concerned with the following specialization of the notion of band of semigroups. Suppose that $I$ is the direct product $J \times K$ of two classes $J$ and $K$. The subsemigroups $S_{\alpha}$ are then described by two subscripts: $S_{i \kappa}(i \in J, \kappa \in K)$. Suppose moreover that $S_{i \kappa} S_{j \lambda} \subseteq S_{i \lambda}$ for all $i, j \in J$ and all $\kappa, \lambda \in K$. We shall then call $S$ a matrix of semigroups of type $\mathcal{T}$. The primary purpose of the present paper is to show (Theorem 4) that a band of semigroups of type $\mathcal{T}$ is a semilattice of semigroups each of which is a matrix of semigroups of type $\mathcal{T}$.

The rest of the paper is devoted to giving necessary and sufficient conditions on a semigroup $S$ that it be a band or a semilattice of (1) simple semigroups, (2) completely simple semigroups, and (3) groups. (Throughout this paper we use the term simple to mean simple without zero, i.e. a simple semigroup is one containing no proper two-sided ideal whatever.) For (1), we have the elegant condition, $a \in S a^{2} S$ for all $a \in S$, due to ${ }^{1}$ Olaf Andersen [1]. If a semigroup $S$ is a class sum of [completely] simple semigroups, it is also a semilattice of [completely] simple semigroups. But a class sum of groups need not be a band of groups, nor need a band of groups be a semilattice of groups; these three categories are characterized by Theorems 6,7 , and 8 , respectively.

We note that a semigroup $S$ is a "band of groups of order one" if and only if each element of $S$ is idempotent. In this case we call $S$ simply a "band," and consequently make the definition: a band is a semigroup every element of which is idempotent. By the same token, we define a semilattice to be a commutative band. A "matrix of groups

Presented to the Society, December 30,1953; received by the editors November 20, 1953.

1 (Added in proof.) This result has also been obtained recently by $\mathrm{R}$. Croisot [8, p. 369]. 
of order one" will be called a "rectangular band." Thus we define a rectangular band to be the direct product $J \times K$ of two classes $J$ and $K$, with multiplication defined by

$$
(i, \kappa)(j, \lambda)=(i, \lambda) \quad(\text { all } i, j \in J ; \kappa, \lambda \in K) .
$$

The reason for the term is the following. Think of $J \times K$ as a rectangular array of points, the point $\alpha=(i, \kappa)$ lying in the $i$ th row and $\kappa$ th column. Then $\alpha=(i, \kappa)$ and $\beta=(j, \lambda)$ are opposite vertices of a rectangle of which the other two vertices are $\alpha \beta=(i, \lambda)$ and $\beta \alpha=(j, \kappa)$.

THEOREM 1. A semigroup $S$ is a band of semigroups of type $\tau$ if and only if there exists a band I and a homomorphism $\phi$ of $S$ onto I such that the inverse image $\phi^{-1}(\alpha)$ of each element $\alpha$ of $I$ is a subsemigroup $S_{\alpha}$ of $S$ of type $\tau$.

Proof. Let $S$ be a band of semigroups $S_{\alpha}(\alpha \in I)$ of type $\mathcal{T}$. Then to each pair of elements $\alpha, \beta$ of the index class $I$ corresponds a unique element $\gamma$ of $I$ such that $S_{\alpha} S_{\beta} \subseteq S_{\gamma}$. Define $\alpha \beta=\gamma$. Since $S_{\alpha} S_{\beta} \cdot S_{\gamma}$ $=S_{\alpha} \cdot S_{\beta} S_{\gamma}$, and $S_{\alpha} S_{\alpha} \subseteq S_{\alpha}$, this multiplication is associative and idempotent, i.e. $I$ becomes a band thereunder. Each element $a$ of $S$ belongs to exactly one $S_{\alpha}$, and, if we define $\phi(a)=\alpha$, the mapping $\phi$ of $S$ onto $I$ is clearly a homomorphism with the property stated in the theorem. The converse is evident.

We shall use the expression, " $S$ is a band $I$ of semigroups $S_{\alpha}(\alpha \in I)$ of type $\mathcal{T}, "$ to indicate the situation described in Theorem 1. $\phi$ will be called the natural homomorphism of $S$ onto $I$.

We shall call a semigroup $S$ simple if $S a S=S$ for every $a \in S$, and completely simple if $S$ is simple and contains a primitive idempotent (cf. Rees [3]). (An idempotent $e$ is primitive if from $f^{2}=f$ and $e f=f e$ $=f$ we conclude $f=e$.) We shall not have occasion to consider semigroups with a zero element, and we consequently omit the phrase without zero. Rees [2] showed (in particular) that every completely simple semigroup is isomorphic with a "matrix semigroup $S$ over a group $G$," defined as follows. Let $J$ and $K$ be any two sets. Let $S$ be the set of all triples $(a ; i, \kappa)$, with $a \in G, i \in J, \kappa \in K$, and let the product of two elements of $S$ be defined by

$$
(a ; i, \kappa)(b ; j, \lambda)=\left(a p_{k j} b ; i, \lambda\right) \quad(a, b \in G ; i, j \in J ; \kappa, \lambda \in K),
$$

where $\left(p_{k j}\right)$ is a fixed $K \times J$ matrix of elements $p_{k j}$ of $G$. Since Suschkewitsch [4] proved essentially the same theorem for finite $S$, we shall refer to this result as the Suschkewitsch-Rees Theorem.

The following theorem is simply a restatement, in the terminology of the present paper, of Theorem 2 of an earlier paper [5]. 
THEOREM 2. A semigroup is a class sum of groups if and only if it is a semilattice of completely simple semigroups.

We remark that if a semigroup $S$ is a class sum of groups, then it is a class sum of mutually disjoint groups. This follows from Theorem 1 of [5], and the evident fact that $S$ admits relative inverses. A similar remark holds if $S$ is a class sum of simple or of completely simple semigroups, the former by Andersen's Theorem (stated below) and the latter by Theorem 2 .

We next express in our present terminology a result due to McLean [6]. Call a band nowhere commutative if $a b=b a$ implies $a=b$.

Theorem (McLean). Every band is a semilattice of nowhere commutative bands.

Since a rectangular band is clearly nowhere commutative, the next theorem sharpens that of McLean.

Theorem 3. Every band is a semilattice of rectangular bands.

Proof. A band is a class sum of groups (of order one), and hence, by Theorem 2, is a semilattice of completely simple bands. Applying the Suschkewitsch-Rees Theorem to a completely simple band $B$, we see that the structure group of $B$ must have order one, and hence the law (2) of multiplication reduces to (1).

We come now to the main result of the present paper.

Theorem 4. A band of semigroups of type $\mathfrak{T}$ is a semilattice of semigroups each of which is a matrix of semigroups of type $\mathcal{T}$.

Proof. Let $S$ be a band $I$ of semigroups $S_{\alpha}(\alpha \in I)$ of type $\mathcal{T}$. By Theorem $3, I$ is itself a semilattice $P$ of rectangular bands $I_{\tau}(\tau \in P)$. Let $\phi$ be the natural homomorphism of $S$ onto $I$, and let $\psi$ be that of $I$ onto $P$. The mapping $a \rightarrow \psi(\phi(a))$ is clearly a homomorphism of $S$ onto $P$. The inverse image $S_{\tau}^{\prime}$ of an element $\tau$ of $P$ is the class sum of all the $S_{\alpha}$ for which $\psi(\alpha)=\tau$, i.e. for which $\alpha \in I_{\tau}$. Describing the elements $\alpha$ of $I_{\tau}$ as pairs $(i, \kappa)$ of elements of index classes $J_{\tau}$ and $K_{\tau}$, as above, then we may write $S_{i \kappa}$ for $S_{\alpha}$ and obtain $S_{i \kappa} S_{j \lambda} \subseteq S_{i \lambda}$ for all $i, j \in J_{\tau}$ and all $\kappa, \lambda \in K_{\tau}$. Thus $S_{\tau}^{\prime}$ is a matrix of semigroups $S_{i \kappa}$, and the latter (being the $S_{\alpha}$ ) are of type $\mathcal{T}$.

Theorem 5. A matrix of [completely] simple semigroups is [completely] simple.

Proof. Let $S$ be a matrix of simple semigroups $S_{i \kappa}(i \in J, \kappa \in K)$. Let $a$ and $b$ be arbitrary elements of $S$; we are to show that there 
exist $x, y$ in $S$ such that $x a y=b$. Suppose $a \in S_{i \kappa}$ and $b \in S_{j \lambda}$. Then $b a b \in S_{j \lambda}$. Since $S_{j \lambda}$ is simple, there exist $u, v$ in $S_{j \lambda}$ such that $u \cdot b a b \cdot v$ $=b$. Hence we may take $x=u b, y=b v$.

Now suppose that the semigroups $S_{i \kappa}$ are completely simple. To show that $S$ is completely simple, it suffices to show that any idempotent element $e$ of $S_{i \kappa}$ is primitive in $S$, as well as in $S_{i \kappa}$. Let $f$ be an idempotent element of $S$ such that $f e=e f=f$. Suppose $f \in S_{j \lambda}$. Then $f e \in S_{j \lambda} S_{i \kappa} \subseteq S_{j \kappa}$ and $e f \in S_{i \kappa} S_{j \lambda} \subseteq S_{i \lambda}$. Hence $S_{j \kappa}$ and $S_{i \lambda}$ must be the same as $S_{j \lambda}$, i.e. $i=j$ and $\kappa=\lambda$. Thus $f \in S_{i \kappa}$, and since $e$ is primitive in $S_{i \kappa}$, we conclude that $f=e$.

From Theorem 5 and the Suschkewitsch-Rees Theorem, it follows that a semigroup is a matrix of groups if and only if it is completely simple.

The following is Theorem 9.5 in [1]. (A proof may be constructed for it by suitably modifying the proof of Theorem 2. $)^{2}$

TheOREM (Croisot-Andersen). The following three assertions concerning a semigroup $S$ are equivalent:

(A) $S$ is a class sum of simple semigroups.

(B) $S$ is a semilattice of simple semigroups.

(C) $a \in S a^{2} S$ for every $a \in S$.

Let us call two elements $a, b$ of a semigroup $S r$-associate ( $l$-associate) if they generate the same right (left) ideal of $S$, and $r \cap l$ associate if they are both $r$ - and $l$-associate. The following is Theorem 7 in [7]:

Theorem (J. A. Green). If an element a of a semigroup $S$ is $r \cap l$ associate with $a^{2}$, then the set of all elements of $S$ which are $r \cap l$-associate with $a$ is a subgroup of $S$.

THEOREM 6. The following four assertions concerning a semigroup $S$ are equivalent:

(A) $S$ is a class sum of completely simple semigroups.

(B) $S$ is a class sum of groups.

(C) $S$ is a semilattice of completely simple semigroups.

(D) $a \in S a^{2} \cap a^{2} S$ for every $a \in S$.

Proof. (A) implies (B), since every completely simple semigroup is a class sum of groups. (B) implies (C) by Theorem 2, and evidently (C) implies (A). (B) implies (D), for, if $a \in S$, then $a$ and $a^{2}$ both belong to the same subgroup $G$ of $S$, so that $a \in G a^{2} \cap a^{2} G \subseteq S a^{2}$ $\cap a^{2} S$. (D) implies (B), for, if $a \in S$, (D) implies that $a$ and $a^{2}$ are

\footnotetext{
2 See footnote 1 .
} 
$r \bigcap l$-associate, and hence $a$ belongs to a subgroup of $S$ by Green's theorem.

THEOREM 7. A semigroup $S$ is a band of groups if and only if it satisfies the following two conditions:

(1) $a \in S a^{2} \cap a^{2} S$ for every $a \in S$;

(2) for every pair of elements $a, b$ of $S, S b a=S b a^{2}$ and $a b S=a^{2} b S$.

Proof. Let $S$ be a band of groups. In particular, $S$ is a class sum of groups, and (1) follows as in the proof of Theorem 6. Let $a, b \in S$. From the fact that $a$ and $a^{2}$ belong to the same subgroup of $S$, and the assumption that $S$ is a band of groups, we conclude that $b a$ and $b a^{2}$ belong to the same subgroup of $S$, and hence $S b a=S b a^{2}$. Similarly, $a b S=a^{2} b S$.

Suppose conversely that (1) and (2) hold in $S$. By (1) and Green's Theorem, $S$ is the class sum of groups $G_{\alpha}(\alpha \in I)$. Let $a, b \in S$. Let $G_{\alpha}$ be the subgroup of $S$ to which $a$ belongs, let $e_{\alpha}$ be the identity element of $G_{\alpha}$, and let $a^{-1}$ be the inverse of $a$ in $G_{\alpha}$. Replacing $b$ by $a^{-1} b$ in the second part of (2), we conclude $e_{\alpha} b S=a b S$. If $a^{\prime}$ is any other element of $G_{\alpha}$, we conclude similarly that $e_{\alpha} b S=a^{\prime} b S$, and hence $a b S=a^{\prime} b S$. Evidently $S a=S a^{\prime}$, and hence $S a b=S a^{\prime} b$. Consequently $a b$ and $a^{\prime} b$ are $r \cap l$-associate. Now, in a semigroup $S$ which is the class sum of groups, two elements of $S$ are $r \cap l$-associate if and only if they belong to the same subgroup of $S$. Consequently $a b$ and $a^{\prime} b$ belong to the same subgroup of $S$. By the left-right dual of this argument, $b a$ and $b a^{\prime}$ also belong to the same subgroup of $S$. Thus the relation of belonging to the same subgroup of $S$ is a congruence relation, whence $S$ is a band of groups.

THEOREM $8 .^{3} A$ semigroup $S$ is a semilattice of groups if and only if it satisfies the following two conditions:

(1) $a \in S a^{2} \cap a^{2} S$ for every $a \in S$;

(2) if $e$ and $f$ are idempotent elements of $S$, then $e f=f e$.

Proof. Assume that $S$ is a semilattice $I$ of groups $G_{\alpha}(\alpha \in I)$. Since $S$ is in particular a class sum of groups, (1) follows as in the proof of Theorem 6. To show (2), let $e$ and $f$ be idempotent elements of $S$. Then $e \in G_{\alpha}$ and $f \in G_{\beta}$ for some $\alpha, \beta \in I$. Since $G_{\alpha}$ and $G_{\beta}$ are groups, $e$ and $f$ must be the identity elements thereof: $e=e_{\alpha}, f=e_{\beta}$. Let $\gamma=\alpha \beta$ ( $=\beta \alpha$ since $I$ is by hypothesis commutative). Let $e_{\gamma}$ be the identity element of $G_{\gamma}$. Since $e_{\alpha} e_{\beta} \in G_{\gamma}$ we have $e_{\gamma} \cdot e_{\alpha} e_{\beta}=e_{\alpha} e_{\beta}$.

Now $e_{\gamma} e_{\alpha} \in G_{\gamma \alpha}=G_{\gamma}$, so that $e_{\gamma} e_{\alpha} \cdot e_{\gamma}=e_{\gamma} e_{\alpha}$. Hence

${ }^{3}$ (Added in proof.) This result has also been noted by R. Croisot [8, p. 375]. 


$$
\left(e_{\gamma} e_{\alpha}\right)^{2}=\left(e_{\gamma} e_{\alpha} \cdot e_{\gamma}\right) e_{\alpha}=e_{\gamma} e_{\alpha} e_{\alpha}=e_{\gamma} e_{\alpha}
$$

Since $e_{\gamma}$ is the only idempotent element of $G_{\gamma}$, we conclude that $e_{\gamma} e_{\alpha}$ $=e_{\gamma}$. Similarly, $e_{\gamma} e_{\beta}=e_{\gamma}$. Hence

$$
e_{\alpha} e_{\beta}=e_{\gamma} e_{\alpha} e_{\beta}=e_{\gamma} e_{\beta}=e_{\gamma} .
$$

Thus $e_{\alpha} e_{\beta}=e_{\alpha \beta}$ for all $\alpha, \beta$ in $I$, whence $e_{\beta} e_{\alpha}=e_{\beta \alpha}=e_{\alpha \beta}=e_{\alpha} e_{\beta}$.

Conversely, let $S$ be a semigroup satisfying conditions (1) and (2). By (1) and Green's theorem, $S$ is a class sum of groups. From Theorem 3 of [5] and condition (2) it then follows that $S$ is a semilattice of groups; moreover, the theorem cited provides an explicit construction for any such $S$.

\section{REFERENCES}

1. Olaf Andersen, Ein Bericht über die Struktur abstrakter Halbgruppen, Thesis Hamburg, 1952, unpublished.

2. D. Rees, On semi-groups, Proc. Cambridge Philos. Soc. vol. 36 (1940) pp. 387400.

3. - Note on semi-groups, Proc. Cambridge Philos. Soc. vol. 37 (1941) pp. $434-435$.

4. A. Suschkewitsch, Über die endlichen Gruppen ohne das Gesetz der eindeutigen Umkehrbarkeit, Math. Ann. vol. 99 (1928) pp. 30-50.

5. A. H. Clifford, Semigroups admitting relative inverses, Ann. of Math. vol. 42 (1941) pp. 1037-1049.

6. David McLean, Idempotent semigroups, Amer. Math. Monthly vol. 61 (1954) pp. $110-113$.

7. J. A. Green, On the structure of semigroups, Ann. of Math. vol. 54 (1951) pp. 163-172.

8. R. Croisot, Demi-groups inversifs et demi-groupes réunions de demi-groupes simples, Ann. Ecole Norm. (3) vol. 70 (1953) pp. 361-379. (Added in proof.)

The Johns Hopkins University 